\title{
Preparing WorldView2 Imagery for Map Updating Projects - Aldjyl/ Aleibayiji (Iraq/ North Baghdad) as a Case Study
}

 \\ Civil Engineering Dept., University of Technology-Iraq, Alsina’a street, 10066 Baghdad, Iraq. \\ *Corresponding author Email: 40004@uotechnology.edu.iq
}

\section{H I G H L I G H T S}



\section{A R T I C L E I N F O}

Handling editor: Wasan I. Khalil

\section{Keywords:}

WorldView2 Image

Pansharpening

Geometric Correction

Controlled Image Based

\begin{abstract}
A B S T R A C T
In the presented study, a WorldView2 WV2 satellite image has been prepared to be used in map updating projects. The performed operations can be classified into two categories, the first category includes pansharpening methods for creating a new 8- spectral bands of WV2 Imagery, each one of them has a 0.5meter spatial resolution. The Pansharpening technique has been implemented with four different approaches, i.e. (Principal Components PC, Wavelet Transform WT, Hyperspherical Color Space HCS, and High Pass Filtering HPF). For these four methods and based on a visual comparison among the quality of the resulting images (with respect to the original Multi-spectral Worldview2 MS-WV2 image), the HCS image has been determined as the best one of them. Because of the high-security situation of the study area, and the impossibility of using the Global Positioning System GPS and other surveying devices there, the Controlled Image Based CIB of 1-meter resolution has been depended on as a source of reference Ground Control Points GCPs. Initially, set GCPs and Check Points are determined on both the HCS Pansharpened image and the CIB image. For maintaining the linearity of the geometric distortion within WV2 original image, the 1st order polynomial transformation model has been selected to perform the geometric correction process. It is clearly concluded that the resulted Root Mean Square Error RMSE of the geometric correction process is acceptable for a wide range of mapping applications. Moreover, the resulting geometrically corrected pan-sharpened WV2 image can be utilized for increasing the interpretation capability of the land features by giving more false color displaying options, and finally supporting the related map updating projects.
\end{abstract}

\section{Introduction}

The motivation behind conducting this study lies in the importance of obtaining a geometrically corrected satellite image with optimizing different parameters of it, such as; spatial resolution, spectral resolution (number of spectral bands) and the Root Mean Square Error RMSE (compared with the real map coordinate system). This resulted in the satellite image, which is to be used as a background for drawing (tracing procedure) new land features, as a fundamental requirement of map updating projects.

The Worldview 2 satellite imagery will be investigated in this study, the technical specification of the satellite, sensor, and image are fully described in $[1,2]$. While, the main applications of such as high-resolution satellite imagery in the map production and updating of land use, land cover, environment, and urban planning are the subject of [3,4].

For the current study, obtaining the geometrically corrected version of the WorldView2 satellite image (with multiple spectral bands and high spatial resolution) requires two types of digital operations, the first is related to obtaining one image of high-spatial resolution and multi-spectral from two images, the first image has high-spatial resolution $(0.5 \mathrm{~m})$ and mono 
panchromatic spectral band. While the second image has a low spatial $(2 \mathrm{~m})$ resolution and eight spectral bands (within the visible and infrared spectrum), this technique is known by different terms, such as image-fusion, spatial merge, or the more common term (Pan sharpening).

The second type of operation that is required to be implemented in the resulted spatial merged satellite image relates to the geometric correction. According to the geometric correction process, the input satellite image will be resampled in a manner that achieves maximum possible geometric accuracy (resulting in a minimum value of RMSE). For this purpose, a set of previously known coordinate points (Ground Control Points GCPs) are to be determined on both the satellite image and their corresponding land positions.

The positioning of the GCPs can be conducted by using different approaches, such as using surveying instruments (GPS, Total Stations), accurate high scale maps or highly geometric corrected satellite images.

There are some difficulties or limitations in both types of digital operations required on the satellite image referred to above. In Pansharpening operations and because the work has been conducted on the whole worldview 2 scenes (in terms of the ground cover and the 8 spectral bands), yielding a new image with too big storage space (more than 20 GigaBytes). Undoubtedly dealing with such as very big data requires using computers with somewhat advanced specifications for ensuring that the work will be implemented without computer hanging or during not long-time duration. With regard to the difficulties of the second type of digital operations (Geometric Correction), it was not possible to locate the GCPs in the traditional way (by using GPS and Total Stations), due to the presence of security limitations, represented by the occurrence of the study area near military areas and other unstable security areas. This led to the search for an alternative approach for locating the Ground Control Points, this approach is the so-called (Controlled Imaged Based CIB), which has not previously been used in relevant research and project studies within Iraq.

The output multi-spectral image that resulted from the pansharpening process is to be used for feature extraction and supporting map updating projects, by using different sets of color composite displaying options, for instance, this approach can be implemented for easily recognizing the water bodies or wetlands from the vegetated area within a rural area.

\section{Theoretical Background}

\subsection{Pansharpening Methods}

The Pansharpening process is a pixel-based fusion technique, it is used widely in the remote sensing field to increase the spatial resolution of the satellite multispectral image. Generally, pansharpening methods increase the spatial resolution of the output image and simultaneously preserve the spectral content in the multispectral image [5]. It is well known that pansharpening is also known as resolution merge, or other names, such as image integration or multisensory data fusion. Different pan-sharpening methods have been extensively discussed in the literature $[5,6,7]$.

In the current study, four of pansharpenig methods adopted by the ERDAS Imagine 2014 software package have been selected for implementation, these methods can produce an output image with more than 4 bands (specifically 8 bands). These methods are not well known in the common Remote Sensing and GIS software packages, for instance, the famous ArcGIS software package implements the pansharpening process by using five methods, all of these methods cannot produce an output image with more than 4 bands [8].

These four pansherpening methods are the Principal Components PCs, Wavelet Transform WT, Hyperspherical Color Space HCS, and High Pass Filtering HPF. A brief description for each method is presented below, as follows:

\subsubsection{Principal Components PC}

In the Principal Components PC based pansharpening method, firstly the PC transform of the Multispectral image is performed, after then, the first PC will be replaced by a Panchromatic band of high spatial resolution image. The final output image is obtained by implementing the inverse PC transform figure 1 illustrates the main sequence of operations required for implementing the PC based pansharpening method $[9,10]$.

\subsubsection{Wavelet Transform WT}

This pansharpening method is based on the wavelet decomposition of the input image. According to the WT original image is separated into different components. Generally, Wavelet Transforms are performed on both MS images as well as PAN images for extracting the low-frequency component of the MS image and extracting the high-frequency component data from the PAN image. The next step is performed by combining these above components to create the fused wavelet coefficient $[9,10]$. Figure 2 summarizes the required operations in this method.

\subsubsection{Hyperspherical Color Space Hcs}

This method has been designed specifically for Worldview 2, it is claimed that the HCS pansharpening method is the best for WV2 compared with the other methods $[11,12]$.

At HCS the multi-spectral intensity component is replaced with an intensity that matched with the panchromatic band, this will provide a sharp image, the overall operations including in this approach have been illustrated in figure 1, other related details concerning the HCS pansharpening method have been extensively explained in [12]. 


\subsubsection{High Pass Filtering HPF}

The Pan Sharpening with high-pass filtering was introduced firstly by Schowengerdt in 1980 and was further adapted by Chavez, Sides, and Anderson in [13]. This approach reduces data quantity and at the same time, it increases the spatial resolution, this is enhancement mechanism is implemented by injecting spatial information of the panchromatic band in multispectral bands, with performing a small convolution window to the panchromatic for reducing the lower-frequency spectral information content. After then, the resulting filtered image will be averaged with Multispectral bands to increase its brightness [13]. Figure 3 shows the overall operations that are required to perform HPF based pansharpening.

\subsection{Geometric Correction}

\subsubsection{An overview}

The Geometric distortion is an error with a satellite image between the recorded image coordinates and its corresponding actual ground coordinates $[14,15]$. These geometric distortions can be classified into two types, the first type concerning the internal distortions that are resulting from the geometry of the sensor. While the second type is related to the external distortions due to the attitude of the sensor or the shape of the ground object [16]. Figure 4 illustrates a simplified overall block diagram of the geometric correction process. More theoretical details about the geometric correction of satellite images by using the polynomial transformation model can be seen in [9].

\subsubsection{The Controlled Image Based}

The Controlled Image Base CIB is a seamless database of digital ortho-mosaic images. CIB has a Ground Sample Distance GSD of one-meter spatial resolution and is orthorectified by using NGA Digital Terrain Elevation Data of Level 2 (DTED Level 2). From a historical point of view, the CIB started as a product was called Arc Digital Raster Image ADRI. This product was based on SPOT imagery, which had a Ground Sampling Distance of 10 meters. This was a huge ortho-mosaic and was difficult to be used with the computer capacities of the times. This product had been converted into a Raster Product Format RPF and was named CIB. The format specifications are called MIL-STD-2411. After the inception of CIB 10m, the users demanded a product that has a Ground Sampling Distance of 5-meter spatial resolution. This became the standard product of CIB, and the CIB with 10 meters became obsolete. Currently, the commercial satellites are imaging the Earth Globe with or better than the 1-meter GSD. NGA now produces CIB with a GSD of 1-meter spatial resolution. This product has high demand by Mission Planners. However, the product of CIB with a 5-meter spatial resolution is still producing [17].



Figure 1: Principal Components Pansharpening [10]

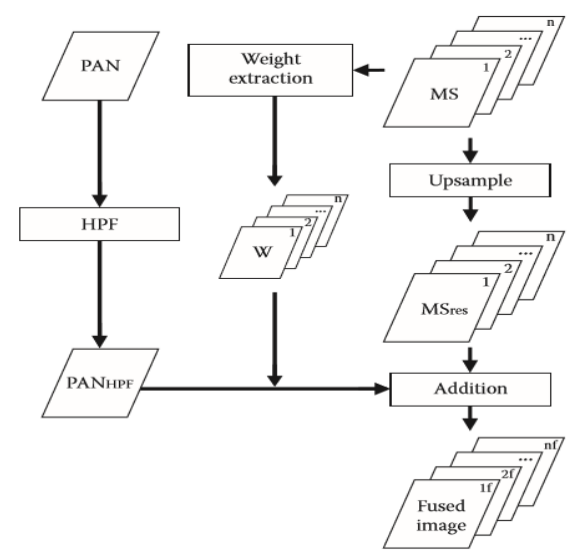

Figure 3: HPF Pansharpening, source [10]



Figure 2: Wavelet Transform Pansharpening [9].

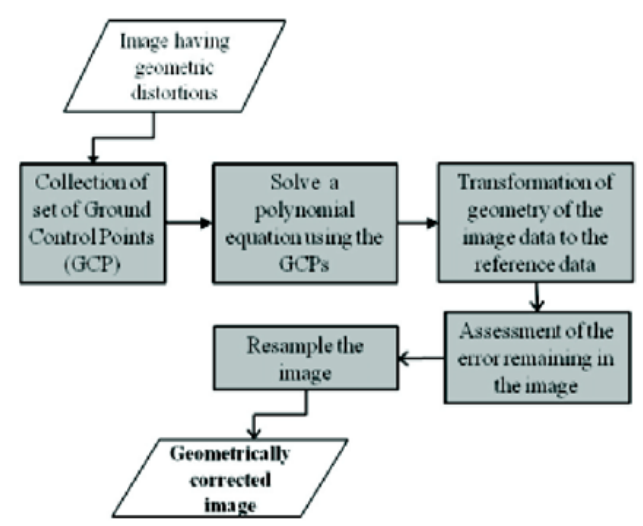

Figure 4: geometric correction steps [18] 
CIB is a grayscale product; the CIB images are projected onto the Arc System, orthorectified, and arranged into a mosaic. The imagery, after then are to be segmented into individual frames and stored as a seamless database per MIL-STD-2411. After performing data compression, an NITF wrapper is written to the frame files. The reference specifications are MIL-STD2411-1, MIL-STD-2411-2, and MIL-STD-2500A [17]. The horizontal accuracy of CIB images depends on the accuracy of the image source from which the CIB had been derived and the accuracy of the Digital Elevation Model (DEM) that is used to orthorectify the image. The Circular Error @ 90 percent CE 90 or the horizontal accuracy is reported within the CIB image frame files. CIB accuracies of about 25 meters are achievable for imagery of $10 \mathrm{~m}$ GSD or better. For $90 \%$ of the randomly chosen points that have been selected for accuracy evaluation [17]. Generally, the CIB products are used by mission planners, FEMA, and in cockpit displays. Moreover, it has been used for feature extraction and imagery registration purposes. So that, the CIB can be considered an important foundation product, especially, in regions that are difficult to be accessed [17].

\section{Methodology}

\subsection{Study Area}

The study area is within Aldjyl/ Aleibayijiregion, it is located in the sharing area between Baghdad and Salahadden governorates of Iraq (figure 5-a), The area is bounded between latitudes $33.72^{\circ}$ and $33.85^{\circ}$ North and longitudes $44.25^{\circ}$ and $44.45^{\circ}$ East, more details of overall features of the study area are shown in figure 5-b.

\subsection{Used Data}

\subsubsection{WorlView2 (WV2) Image}

A WorlView2 (WV2) image (figure 6) has $8 \mathrm{MS}$ bands of $2 \mathrm{~m}$ spatial resolution and a single PAN band of $0.5 \mathrm{~m}$ spatial resolution. According to the metadata associated with the original NTIF image format, the acquisition date of the image is 17March-2017 as shown in figure 7.

\subsubsection{Controlled Image Based (CIB)}

A CIB for the study area (figure 8) has been adopted as a reference of the Ground Control Points (GCPs).

\subsection{The Processing Steps}

The ERDAS Imagine 2014 software package has been adopted to implement the current study, the processing steps have been arranged and illustrated as shown in figure 9. These four methods, i.e., Wavelet Transform, Principal component, Hyperspherical Color Space, and High Pass Filtering respectively.
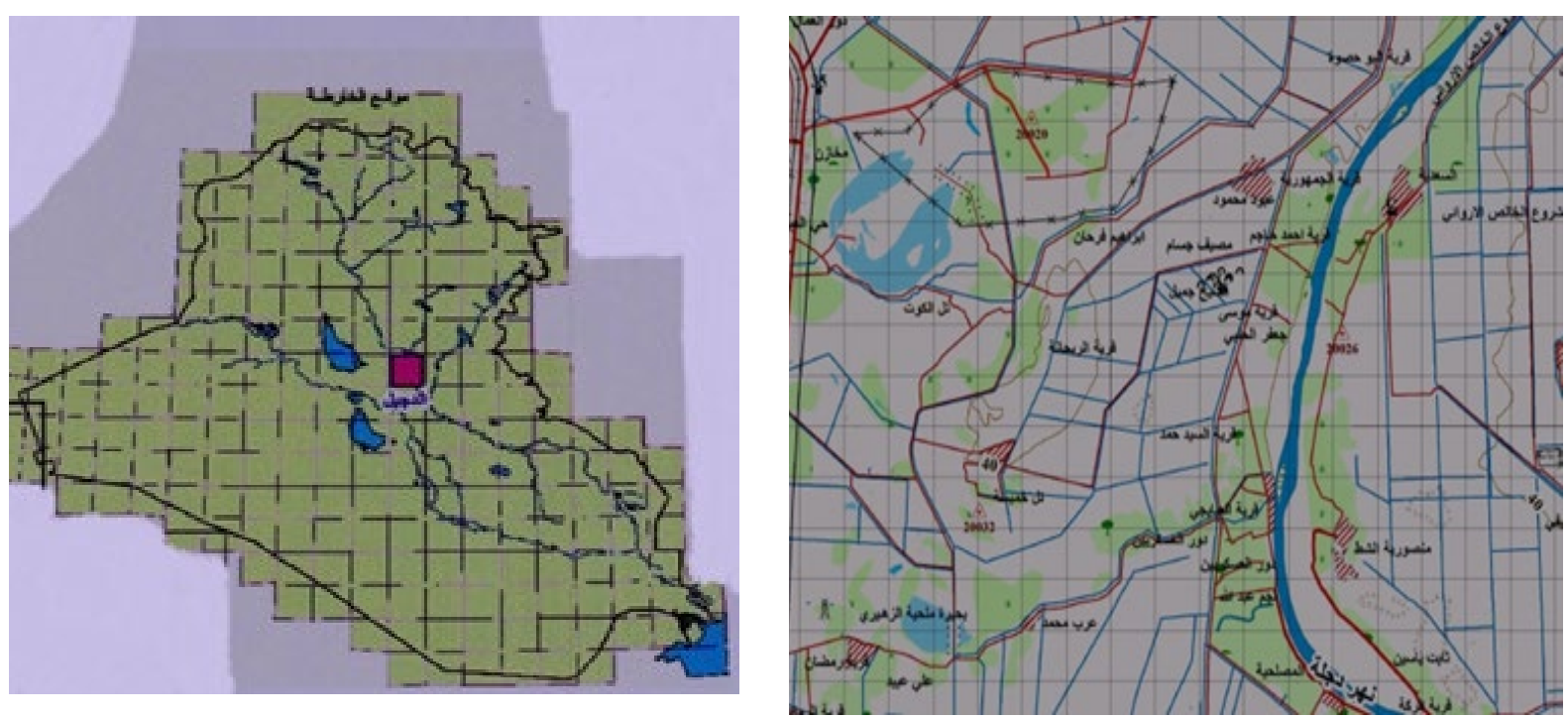

Figure 5: (a) study area relative to Iraq country, (b) topographic map of the study area 


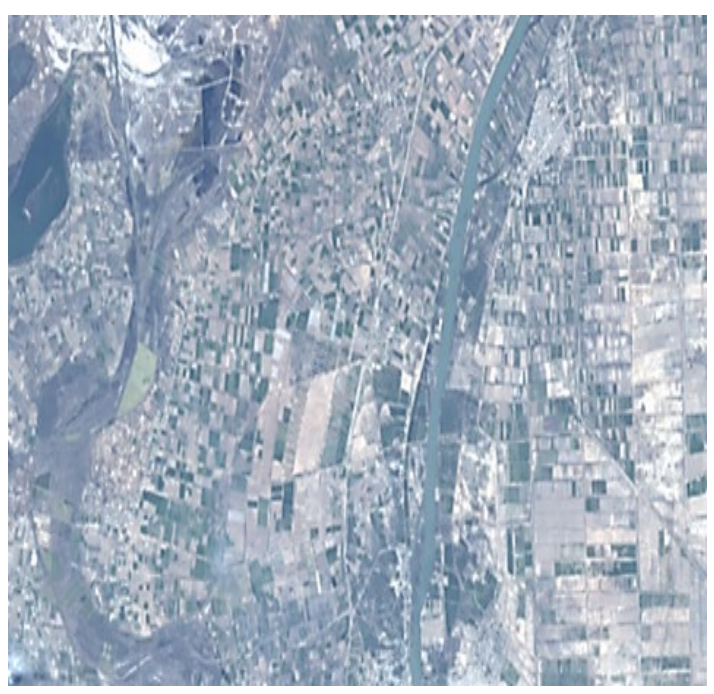

Figure 6: WV2 image of the study area

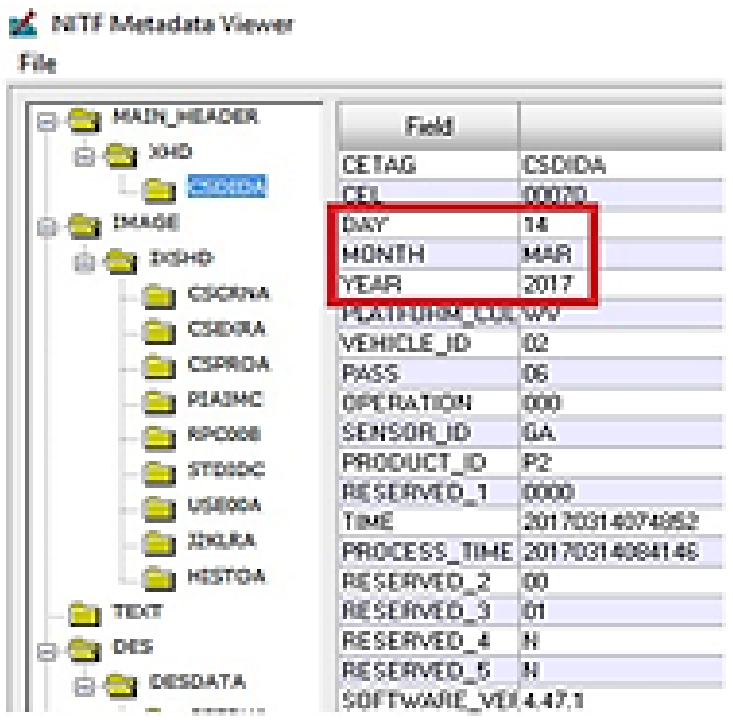

Figure 7: 7 Date of Acquisition for the used



Figure 8: CIB for the study area

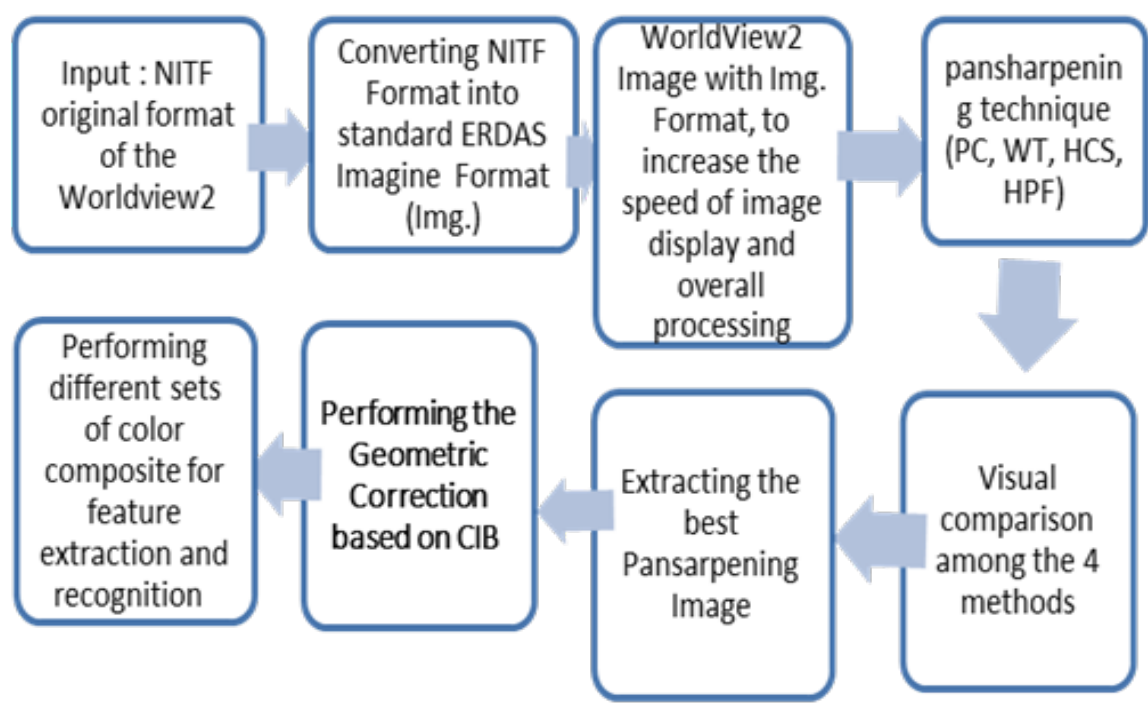

Figure 9: block diagram of the processing steps 


\section{Results and Discussion}

\subsection{The pansharpening methods}

The four pansharpening methods have been implemented on the input WV2 image, figure 10 (a, b, c, and d) illustrates the resulted output pansharpening images.

\subsection{Comparison of Methods}

Based on subjective criteria (visual quality of the output images), it is clear that the HCS method is the best of the four methods as shown in figure 10, the traditional PC method gives the worst result, because of the non-color balance between it and the original multispectral WV2 image. For the Wavelet based method, it is clear that its output image has very good color matching with the original multispectral WV2 image, but the appearance of the ground features has a noticeable pixelling effect. Finally, the HPF method has produced an overly bright image.

\subsection{Color Composite}

The output 8-bands imagemap with its $0.5 \mathrm{~m}$ spatial resolution gives many options in displaying the pan-sharpened image map, this will certainly increase the interpretation capability of the WV2 image, especially in separating between the water bodies and the vegetated areas as shown in figure 11, in this figure, Different color composite images can be utilized for obtaining maximum feature extraction and for increasing the interpretation capability of the WV2 image. Figure 11 illustrates two sets of color composite, the first is true color (RGB 532), while, the second, is false color, replacing the red band by the infrared, which is optimum for recognition between vegetation areas and water bodies.

\subsection{Geometric Correction}

Set of GCPs (34 GCPs) are determined on both the CIB and HCS pansharpened image as shown in figures 12 and 13 respectively. Some of these GCPs have been set as Check Points as shown in table 1. The UTM map projection (Zone $38 \mathrm{~N}$ ) and WGS 84 horizontal datum with its WGS 84 ellipsoid have been selected as cartographic-geodetic parameters that are required in the geometric correction process. The first order polynomial model has been selected as the transformation model between the input and output coordinate systems. The numerical values of X-Residual, Y-Residual, RMSE, Total X-Error, Total Y-Error, and Total RMSEfor both GCPs and Check Points have been recorded in table 2.

Generally, such values have acceptable tolerance in mapping projects with a scale of not more than 1:10000. The resulting geometrically corrected WV2 image map of this study is illustrated in figure 14.
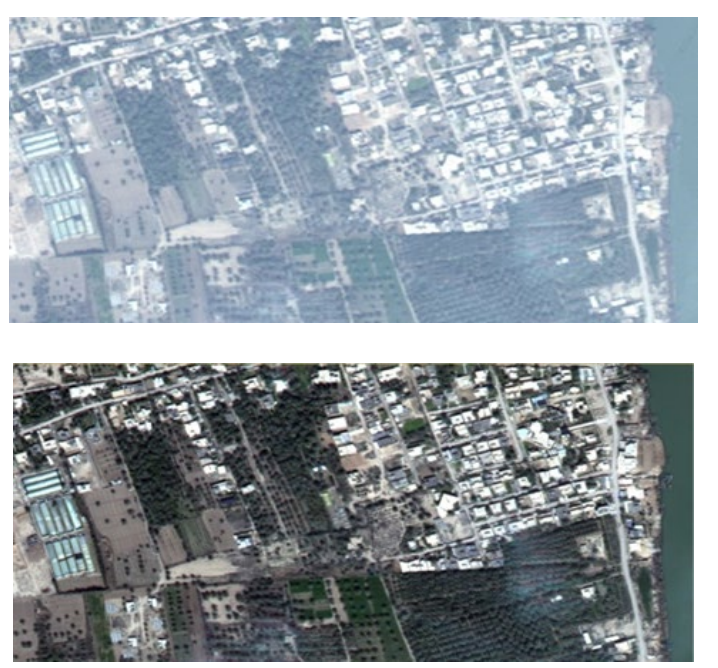


Figure 10: (a) Wavelet Transform WT (b) Principal Components Transform PCT (c) Hyperspherical Color Space HCS (d) High Pass Filtering HPF


Figure 11: (a) True color composite (RGB 532), (b) -False color composite (RGB 632) 


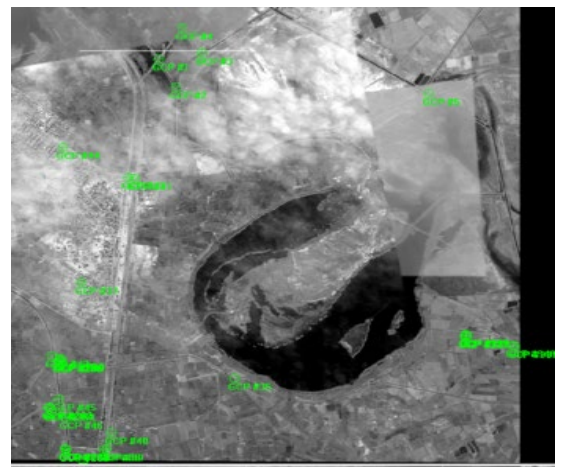

Figure 12: GCPs on CIB



Figure 13: GCPs on HCS pansharpened image

Table 1: Input and reference coordinates of the GCPs

\begin{tabular}{|c|c|c|c|c|c|}
\hline & X-Input (m) & Y-Input (m) & X-Ref. (m) & Y-Ref. (m) & GCP- \\
\hline GCP \#1 & 432251.797 & 3745249.908 & 432250.043 & 3745251.035 & Control \\
\hline GCP \#2 & 432460.222 & 3744863.686 & 432464.360 & 3744866.819 & Control \\
\hline GCP \#3 & 432764.608 & 3745333.480 & 432767.513 & 3745338.761 & Control \\
\hline GCP \#4 & 432537.380 & 3745683.920 & 432536.470 & 3745688.278 & Check \\
\hline GCP \#5 & 435481.647 & 3744762.901 & 435479.233 & 3744767.990 & Control \\
\hline GCP \#35 & 433157.993 & 3740799.025 & 433161.582 & 3740798.837 & Control \\
\hline GCP \#37 & 431332.971 & 3742141.235 & 431335.283 & 3742143.739 & Control \\
\hline GCP \#38 & 431595.161 & 3739812.765 & 431598.251 & 3739816.399 & Check \\
\hline GCP \#39 & 431625.682 & 3739811.983 & 431630.269 & 3739812.200 & Control \\
\hline GCP \#40 & 431689.825 & 3740046.657 & 431691.681 & 3740044.199 & Control \\
\hline GCP \#41 & 431964.241 & 3743595.415 & 431966.720 & 3743596.095 & Control \\
\hline GCP \#42 & 431894.214 & 3743603.344 & 431897.960 & 3743608.168 & Check \\
\hline GCP \#44 & 431110.767 & 3744019.786 & 431113.520 & 3744020.575 & Control \\
\hline GCP \#43 & 430977.325 & 3741124.920 & 430979.515 & 3741124.250 & Control \\
\hline GCP \#45 & 431058.269 & 3740513.723 & 431063.568 & 3740516.709 & Control \\
\hline GCP \#46 & 431146.729 & 3740258.317 & 431154.907 & 3740260.245 & Check \\
\hline GCP \#275 & 431355.990 & 3739667.775 & 431359.714 & 3739669.793 & Control \\
\hline GCP \#276 & 431340.532 & 3739667.648 & 431343.632 & 3739669.793 & Control \\
\hline GCP \#279 & 431133.869 & 3739822.581 & 431138.486 & 3739823.653 & Control \\
\hline GCP \#280 & 431145.866 & 3739801.637 & 431149.606 & 3739804.574 & Control \\
\hline GCP \#281 & 431149.637 & 3739819.072 & 431153.263 & 3739821.480 & Control \\
\hline GCP \#282 & 430941.205 & 3740395.367 & 430947.247 & 3740397.368 & Control \\
\hline GCP \#283 & 430951.004 & 3740406.452 & 430956.809 & 3740408.234 & Control \\
\hline GCP \#284 & 430965.321 & 3740370.455 & 430971.587 & 3740373.028 & Check \\
\hline GCP \#285 & 431059.823 & 3741053.925 & 431063.729 & 3741054.967 & Control \\
\hline GCP \#286 & 431068.694 & 3741057.998 & 431073.070 & 3741059.568 & Control \\
\hline GCP \#287 & 431083.826 & 3741078.745 & 431089.372 & 3741080.176 & Control \\
\hline GCP \#288 & 431090.209 & 3741065.603 & 431094.670 & 3741066.704 & Check \\
\hline GCP \#335 & 435918.710 & 3741414.244 & 435922.920 & 3741416.582 & Control \\
\hline GCP \#336 & 435932.820 & 3741412.098 & 435937.857 & 3741413.218 & Control \\
\hline GCP \#337 & 435914.882 & 3741393.787 & 435918.868 & 3741396.426 & Control \\
\hline GCP \#338 & 435929.030 & 3741390.296 & 435933.721 & 3741393.296 & Check \\
\hline GCP \#340 & 436483.915 & 3741264.326 & 436488.377 & 3741266.634 & Control \\
\hline GCP \#339 & 436503.046 & 3741250.641 & 436506.228 & 3741252.576 & Control \\
\hline
\end{tabular}




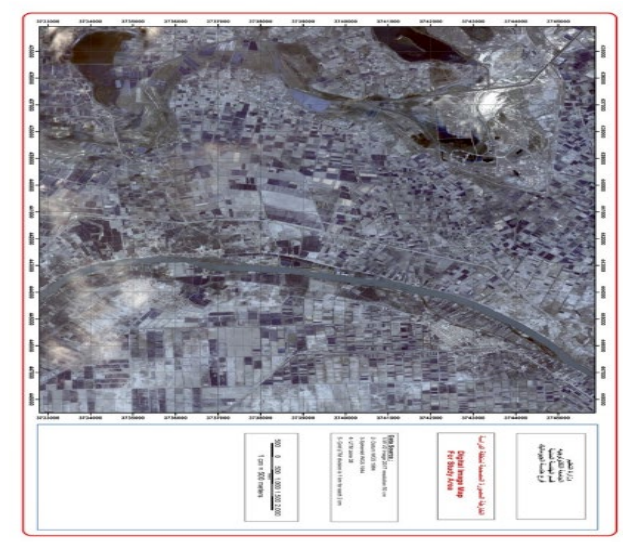

Figure 14: geometrically corrected WV2 image map

Table 2: X-Residual, Y-Residual and RMSE for individual GCPs and Check Points, Total X- Error, Total Y- Error and Total RMSE for GCPs and Check Point

\begin{tabular}{llllllll}
\hline GCP\# & X- & Y-Res. & RMSE & GCP\# & X-Res. & Y-Res. & RMSE \\
\hline GCP \#1 & -2.932 & $1.686-$ & 3.382 & GCP \#276 & $1.539-$ & 1.130 & 1.909 \\
GCP \#2 & 2.727 & 0.403 & 2.756 & GCP \#279 & 0.069 & 0.042 & 0.081 \\
GCP \#3 & 1.791 & 2.365 & 2.967 & GCP \#280 & $0.820-$ & 1.912 & 2.080 \\
GCP \#4 & $1.813-$ & 1.373 & 2.274 & GCP \#281 & $0.923-$ & 1.377 & 1.658 \\
GCP \#5 & $3.822-$ & 1.938 & 4.285 & GCP \#282 & 1.844 & 0.829 & 2.021 \\
GCP \#35 & $0.315-$ & $1.810-$ & 1.837 & GCP \#283 & 1.614 & 0.605 & 1.723 \\
GCP \#37 & $0.802-$ & 0.753 & 1.100 & GCP \#284 & 2,053 & 1.405 & 2.487 \\
GCP \#38 & $1,453-$ & 2.538 & 2.924 & GCP \#285 & 0.115 & $0.344-$ & 0.363 \\
GCP \#39 & 0.042 & $0.884-$ & 0.885 & GCP \#286 & 0.588 & 0.182 & 0.616 \\
GCP \#40 & $2.545-$ & 3.636 & 4.438 & GCP \#287 & 1.771 & 0.033 & 1.772 \\
GCP \#41 & 0.274 & $1.597-$ & 1.620 & GCP \#288 & 0,678 & -0.293 & 0,738 \\
GCP \#42 & 1,547 & 2.553 & 2.986 & GCP \#335 & 0.746 & 0.120 & 0.755 \\
GCP \#44 & 0.792 & $1.488-$ & 1.685 & GCP \#336 & 1.570 & -1.100 & 1.917 \\
GCP \#43 & -1.560 & 2.064 & 2.587 & GCP \#337 & 0.509 & 0.428 & 0.665 \\
GCP \#45 & 1.177 & 1.761 & 2.118 & GCP \#338 & 1,213 & 0.786 & 1.446 \\
GCP \#46 & 3,899 & 0.765 & 3.973 & GCP \#340 & 0.917 & 0.050 & 0.918 \\
GCP & 0.915 & 1.001 & 1.356 & GCP \#339 & -0.370 & $0.321-$ & 0.490 \\
For the GCPs & Total X-Error (m) & Total Y-Error (m) & Total RMSE (m) \\
For the Check & 1.5406 & Total X-Error (m) & 1.3993 & Total Y-Error (m) & Total RMSE (m) \\
Points & 2.041 & 1.6083 & & 2.5979 & \\
\end{tabular}

\section{Conclusions}

1) Generally, the pansharpening methods for satellite images with high spatial and spectral resolutions, such as WV2 images are time consuming operations, so that it is highly recommended to use computers with high specifications in terms of CPU and RAM. Intel Core i7 with $2.4 \mathrm{GHz}$ for CPU and $16 \mathrm{~GB}$ of RAM.

2) For the pansharpening methods, the traditional PC method gives the worst result. While the best image has resulted from the HCS method, this is agreed with [12].

3) The pansharpenend image that is resulted from Wavelet based method has very good color matching with the original multispectral WV2 image, but the appearance of the ground features has a noticeable pixelling effect.

4) The HPF pansharpening method produces an over the bright image (compared with the original WV2 multispectral image).

5) The original circular horizontal error of the used WV2 image is about 6 meters.

6) For a region with a security restriction of using GPS and other surveying devices, the CIB can be adopted to obtain an adequate imagemap and subsequent other thematic maps with a spatial horizontal accuracy of 1 meter.

Based on the $0.5 \mathrm{~mm}$ plotting tolerant standard in mapping applications, the geometric accuracy of the produced Imagemap is acceptable with the scale not exceed than 1:10000. The output 8-bands image with its $0.5 \mathrm{~m}$ spatial resolution gives many options in displaying the pan-sharpened image; this will certainly increase the interpretation capability of the WV2 image, especially in separating between the water bodies and the vegetated areas. This will certainly increase the interpretation capability of the WV2 image, especially in separating between the water bodies and the vegetated areas.

Author contribution

All authors contributed equally to this work. 


\section{Funding}

This research received no specific grant from any funding agency in the public, commercial, or not-for-profit sectors.

Data availability statement

The data that support the findings of this study are available on request from the corresponding author.

Conflicts of interest

The authors declare that there is no conflict of interest.

\section{References}

[1] DigitalGlobe, Inc. 2010, Radiometric Use of WorldView-2 Imagery, Technical Note, Release date: 1 November (2010).

[2] R. Zhang, M. Zeng and J. Chen, Study on geological structural interpretation based onWorldview-2 remote sensing image and its implementation, Procedia Environ. Sci., 10 (2011) 653-659. https://doi.org/10.1016/j.proenv.2011.09.105

[3] S.D.Jawak and A.J.Luis, A spectral index ratio-based Antarctic land-cover mapping using hyperspatial 8-band Worldview2 imagery, Polar. Sci., 7(2013) 18-38. https://doi.org/10.1016/j.polar.2012.12.002

[4] O.Mutanga, E. Adam and M.A. Cho, High density biomass estimation for wetland vegetation using Worldview-2 imagery and random forest regression algorithm, Int. J. Appl. Earth Obs. Geoinf., 18(2012) 399-406. https://doi.org/10.1016/j.jag.2012.03.012

[5] C. Pohl and J.L Van Genderen, Multisensor image fusion in remote sensing: concepts, methods and application, Int. J. Remote Sens., 19 (1998) 823-854. https://doi.org/10.1080/014311698215748

[6] N. Ouarab, M. Achir, N. Khettal and Y. Smara, Spatial enhancement of satellite images using data fusion algorithms in the wavelet domain, Proceedings of the IEEE/ISPRS joint workshop on remote sensing and data fusion over urban areas, (2001) 293-298.

[7] V. Vijayaraj, A Quantitative Analysis of Pansharpened Images, MSc. Thesis, Mississippi State University, Mississippi, (2004).

[8] ArcGIS Desktop10.7.1 Software Package, ESRI Inc., (2019).

[9] Intergraph Corporation; ERDDAS Field Guide; (2013).

[10] Christine Pohl John, van Genderen, Remote Sensing Image Fusion: A Practical Guide, (2017) by Taylor Francis Group, LLC.

[11] DigitalGlobe, https://www.yumpu.com/en/document/read/11251391/worldview-2-imagery-basics-and-erdas-imagine.

[12] C. Padwick, M. Deskevich, F. Pacifici, and S. Smallwood, WorldView-2 Pan-Sharpening, ASPRS 2010 Annual Conference, San Diego, California. April (2010) 26-30.

[13] A. Ghosh, Assessment of pan-sharpened very high-resolution WorldView-2 images, Article in International Journal of Remote Sensing · December (2013).

[14] T. M. Lillesand and R. W.Kiefer, Remote Sensing and Image Interpretation, Fourth Edition, John Wiley and Sons Inc, Singapore, (2003).

[15] P. R. Wolf and B. A. Dewitt, Elements of Photogrammetry with Applications in GIS, Third Edition, McGraw Hill, USA, (2000).

[16] W. K. Pratt, Digital Image Processing, John Wiley and Sons, New York (1978).

[17] NATIONALGEOSPATIAL-INTELLIGENCEAGENCY.

[18] https://www.researchgate.net/figure/Steps-in-geometric-correction-process_fig7_319963167. 